Rapid Reviews COVID-19

\title{
Review 1: "Factors Driving Extensive Spatial and Temporal Fluctuations in COVID-19 Fatality Rates in Brazilian Hospitals"
}

\section{Francisco Benita ${ }^{1}$}

\author{
${ }^{1}$ Singapore University of Technology and Design
}

Published on: Jan 26, 2022

License: Creative Commons Attribution 4.0 International License (CC-BY 4.0). 


\section{$\underline{\text { RR:C19 Evidence Scale rating by reviewer: }}$}

- Reliable. The main study claims are generally justified by its methods and data. The results and conclusions are likely to be similar to the hypothetical ideal study. There are some minor caveats or limitations, but they would/do not change the major claims of the study. The study provides sufficient strength of evidence on its own that its main claims should be considered actionable, with some room for future revision.

$* * * * * * * * * * * * * * * * * * * * * * * * * * * * * * * * * * * * * * *$

\section{Review:}

This preprint examines weekly COVID-19 in-hospital mortality rates of 14 (out of 27) state capitals in Brazil. The focus is on the rise and expansion of the Gamma variant over the period from January 2020 to July 2021. Authors track death rates throughout healthcare pressure indicators of personnel (nurses, nurse assistants, physiotherapists, physicians and critical care specialist) and equipment (critical care beds, ICU beds, ventilators). A Bayesian model is used to compare these indices against fatality rates. Results are presented by location and age group (0-15, 16-29, 30-39, 40-49, 50-59, 60$69,70-74,75-79,80-74,85-59$ and 90+). Findings suggest that the marked increase in fatalities (particularly among adults 50+) is better explained by changes in healthcare pressures rather than the direct effect of the Gamma variation. Lastly, it is argued that in-hospital fatalities in the 14 cities could have been reduced, in the absence of pandemic resource limitations, by around $30 \%$.

While there is a lot that is potentially interesting in this preprint, and it is clearly informative, the spatial aspect of the methodological framework is misleading as there is no spatial component involved. Results are only presented by city and hypothetical scenarios evaluated using a baseline across locations.

The work is rigorous, and I appreciate the effort in documenting the evolution of inhospital mortality for selected state capitals but its overall significance at the national or state level remains unclear. For example, selected cities may concentrate a large proportion of the population (not mentioned anywhere in the manuscript) but fatalities together represent only $20 \%$ of the total number of deaths over the analysed period. When possible, the paper could be strengthened by further presenting a comparative analysis among the remaining excluded municipalities. This is something authors may consider addressing because non-pharmaceutical interventions are usually 
implemented by state and not by city. It is also claimed that "the impact of Gamma in Brazil's hospitals has predominantly been indirect and mediated through pre-existing geographic inequities". However, 14 locations (e.g., cities) is a small sample size to think about a geographic phenomena.

Lastly, Figure 3(B) could be improved by using a different color code, say gray color, for not statistically significant correlations. In this way, readers will more rapidly identify relevant healthcare pressure indicators associated with age-standardised inhospital fatality rates. 